\title{
Observations of the Antarctic infrared sky spectral brightness
}

\author{
Jon S. Lawrence*, Michael C. B. Ashley, Michael G. Burton, and John W.V. Storey \\ School of Physics, University of New South Wales
}

\begin{abstract}
An important parameter that defines the effectiveness and efficiency of any optical or infrared sky survey is the atmospheric character of the observing site. Of prime importance is the sky spectral brightness, which determines the sensitivities and the observing time required to complete a particular survey. This paper presents observations of the near-infrared sky spectral brightness measured at the South Pole throughout the 2001 winter with an automated instrument, the Near Infrared Sky Monitor (NISM). Results from the NISM confirm that the South Pole sky spectral brightness is up to two orders of magnitude lower than at any other ground-based site, consistent with previous observations. These results indicate that the Antarctic plateau is an ideal place to site a future infrared sky survey telescope.
\end{abstract}

Keywords: Antarctic astronomy, site-testing, radiative transfer, atmospheric effects

\section{INTRODUCTION}

An extensive site-testing campaign has been conducted at the South Pole over the last decade in order to determine the applicability of the Antarctic atmosphere for astronomical observations. Instrumentation has been designed to measure the infrared sky background, the atmospheric turbulence profiles, and the sub-millimetre opacity. These experiments have shown that the sky spectral brightness in the near ${ }^{1}$ and mid $^{2}$ infrared is significantly lower than observed at any other ground-based site.

The lowest infrared Antarctic sky emission will occur for wavelengths that lie on the Wien side of the Planck function and wavelengths for which airglow emission is lowest or non-existent. Both of these requirements are satisfied for a region of wavelengths in the atmospheric $\mathrm{K}$ window centred around $2.4 \mu \mathrm{m}$. Sky brightness in this window (the $\mathrm{K}_{\mathrm{dark}}$ window) has been utilised in many studies as a representation of site quality.

The current study reports an analysis of the data collected by the Near Infrared Sky Monitor (NISM) over the 2001 season at the South Pole. The NISM, a low-powered, autonomous, site-testing instrument, measures sky spectral brightness in the $\mathrm{K}_{\mathrm{dark}}$ window via a differential detection technique. The motivation for the 2001 operation of the NISM is to verify the robustness of the differential measurement technique and the reliability of the instrument through the Antarctic winter, in order that it can be deployed to more remote locations on the Antarctic plateau (specifically Dome C) in the future.

\section{INSTRUMENT CONFIGURATION}

The NISM is shown in Figure 1 installed on the roof of the Automated Astrophysical Site Testing Observatory (AASTO), a facility derived from the Automatic Geophysical Observatory (AGO) design, that has been in operation at the Amudsen-Scott South Pole station since 1997. The NISM observes in two 4 degree field-of-view beams angularly separated by 45 degrees, which are collimated and alternately detected (via a reflective chopper wheel) with a Stirling cycle cooled single-element InSb detector. The optical board of the NISM is shown in Figure 2. The cooled NISM filter has a centre wavelength of $2.379 \mu \mathrm{m}$, and an optical (FWHM) bandwidth of $0.226 \mu \mathrm{m}$. The detector measures the difference signal between the two apertures. The instrument FOV is scanned in elevation across the sky through 180 degrees and back, every four hours. This represents a skydip, an example of which is shown in Figure 3. A calibration blackbody (an oxygen-free copper cavity coated with high infrared absorption paint) is positioned at one horizon.

*j1@phys.unsw.edu.au; phone 612 9385 5003; fax 6129385 6060; http://www.phys.unsw.edu.au/astro; University of New South Wales, Sydney NSW 2052, Australia 


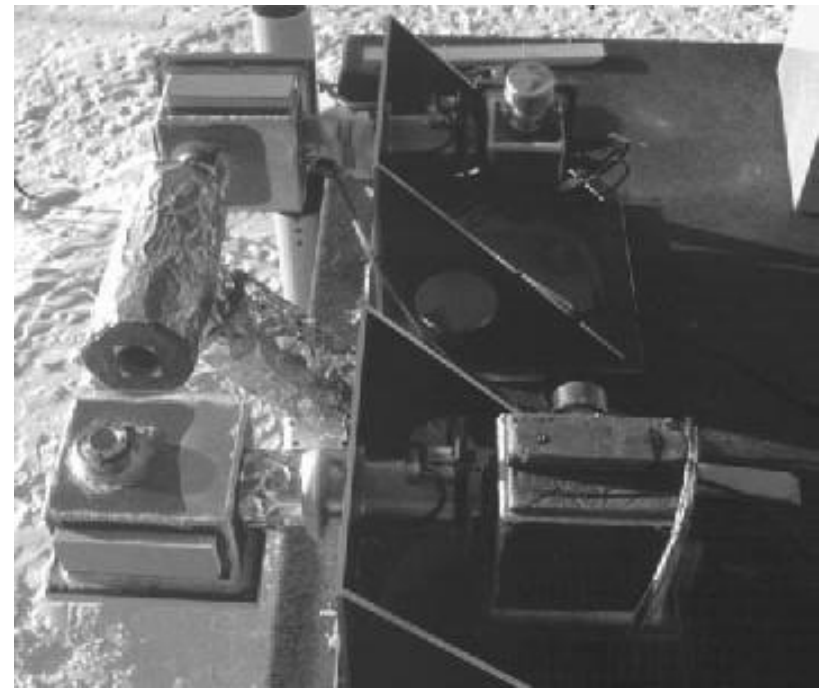

Fig 1: The Near Infrared Sky Monitor (top) and the Mid Infrared Sky Monitor (bottom) installed on the roof of the AASTO at the US Amudsen-Scott South Pole Station. The calibration blackbody is shown between the two instruments.

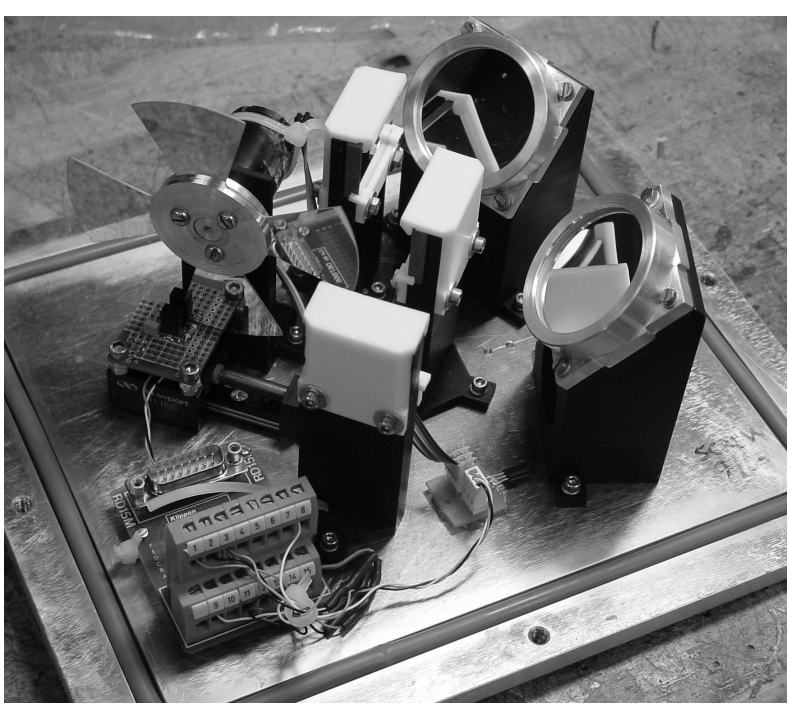

Fig 2: The optical board of the NISM, showing the reflective chopper wheel and differential mirror layout.

The NISM is operated remotely and has a power budget of $\sim 10 \mathrm{~W}$. Data, which include instrumental diagnostics, temperatures of the black body calibrator, and the differential spectral brightness measurement and instrumental noise as a function of instrument zenith angle, are sent via satellite to an email account at the University of New South Wales. For more details of the operation of the NISM (and its companion instrument, the MISM) see refs $(3,4)$.

\section{DATA ANALYSIS}

The sky spectral brightness in the near infrared arises from two principal emission mechanisms: airglow and thermal emission. The airglow occurs due to excitation of $\mathrm{OH}$ molecules in the upper atmosphere boundary layer $(80 \sim 110 \mathrm{~km})$. Thermal emission arises because the troposphere radiates like an optically thin blackbody, to which an effective temperature (which is the convolved atmospheric temperature profile) can be ascribed.

The NISM makes a purely differential spectral brightness measurement; the blackbody calibrator temperature is then used to give a measure of the sky background. Analysis of the 2001 NISM data is described in more detail in ref (4). The procedure requires several factors to determine the zenith sky-spectral-brightness. Firstly, from the instrument skydips, the signal strength at two instrument positions (as indicated in figure 3): $\alpha$, which corresponds to one instrument arm pointing at zenith and the other at $45^{\circ}$, and $\beta$, which corresponds to one arm pointing at $45^{\circ}$ and the other at the blackbody source, is required. Secondly the atmospheric optical depth in the NISM filter window, and the ratio of the airglow flux to atmospheric thermal emission are required: these values are determined from curve fits to NISM skydips. The optical depth is found to have a mean value of $\sim 0.6$, which drops to $\sim 0.2$, implying clear sky conditions and increases to $\sim 1.6$, implying cloudy conditions. For more than $70 \%$ of time, the airglow emission is found to be less than $2 \%$ of the value of the thermal emission. This indicates that the NISM filter used is appropriate for obtaining the lowest sky spectral brightness in the near infrared (because the airglow emission is lowest). Thirdly, several instrumental factors are required (see ref (4)). These parameters combined with the blackbody flux calculated from the blackbody source temperature can then be used to derive a value for the zenith sky-specrtral-brightness. 


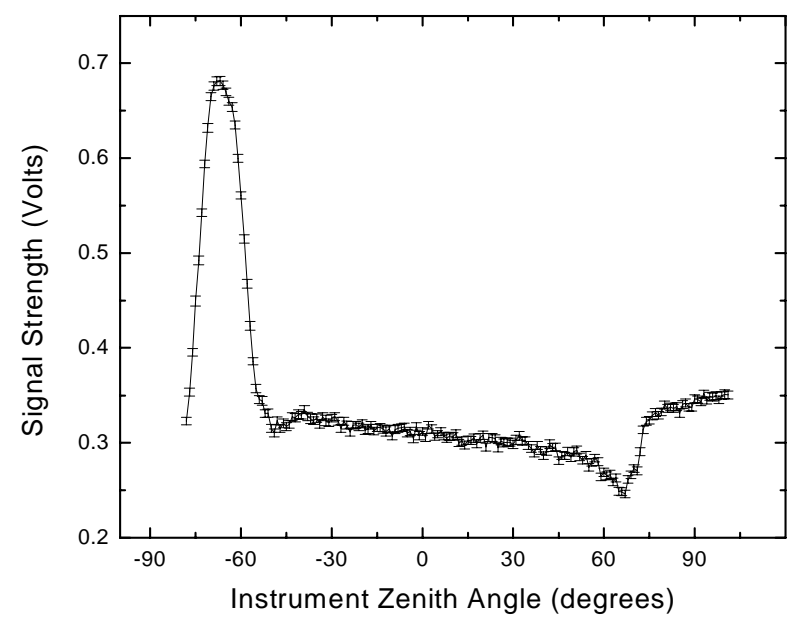

Fig 3: Example of a NISM sky dip, representing a scan of the instrument from horizon to horizon. The peak at $-67.5^{\circ}$ corresponds to one instrument beam pointing to the blackbody calibrator and the other at the sky.

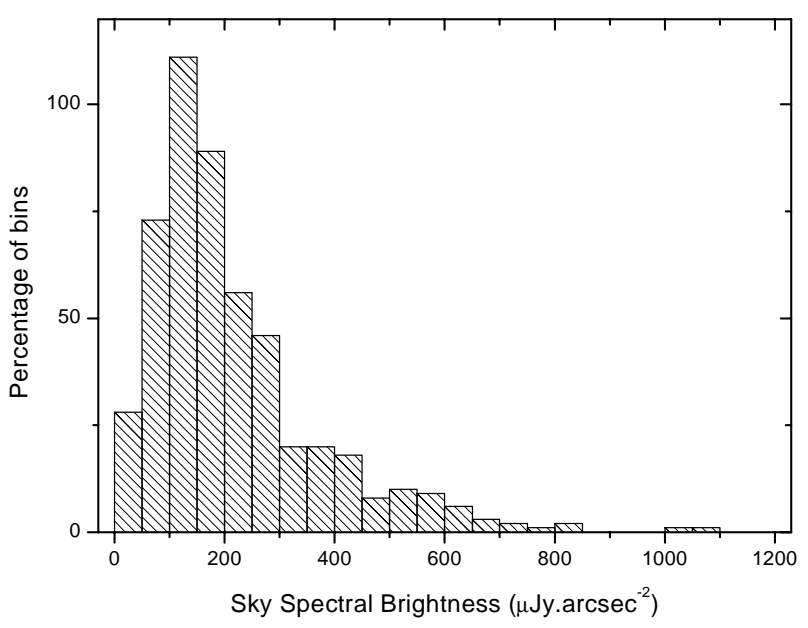

Figure 4. Histogram of the 2001 South Pole winter sky spectral brightness values determined from analysis of NISM sky dips.

\section{RESULTS AND DISCUSSION}

A histogram of the South Pole winter sky-spectral-brightness determined by the NISM is shown in figure 5; the mean value is $220 \mu \mathrm{Jy} \cdot \operatorname{arcsec}^{-2}$. The values for the best $75 \%, 50 \%$, and $25 \%$ of the time are 150,110 , and $80 \mu \mathrm{Jy} \cdot \operatorname{arcsec}^{-2}$ respectively. The sky spectral brightness is consistently below $200 \mu \mathrm{Jy} \cdot \operatorname{arcsec}^{-2}$, and is generally stable over several hours, showing trends determined by slowly varying atmospheric conditions that can last several days. Intermittent periods of very strong atmospheric emission $\left(\sim 1000 \mu \mathrm{Jy} \cdot \operatorname{arcsec}^{-2}\right)$ are also observed. These high brightness values correspond well with periods of high cloud cover (which represent a high optical depth), although the cloud cover statistics are daily approximations from subjective observations only.

Comparisons with other ground-based sites are difficult due to the specific nature of the NISM wavelength filter, which is not commonly used at temperate sites such as Mauna Kea or Chile. Observations with standard $\mathrm{K}$ window filters at Mauna Kea give typical sky spectral brightness values of $4000 \mu \mathrm{Jy} \cdot \operatorname{arcsec}^{-2}$ for the darkest sky conditions. Rebinning of narrow resolution sky spectra gives a rough estimate of the Mauna Kea spectral brightness at $K_{\text {dark }}$ of $\sim 1000-2000$ $\mu \mathrm{Jy} \cdot \operatorname{arcsec}^{-2}$, which is 10-40 times the brightness of the best South Pole conditions. A comparison of South Pole to Mauna Kea sky brightness in the near and mid infrared is shown in figure 5.

Balloon borne observations of the near infrared sky spectral brightness taken at different locations give values that differ by a significant amount and range from 30-130 $\mu \mathrm{Jy} \cdot \operatorname{arcsec}^{-2}$. This fluctuation is consistent with the highly variable sky brightness measurements observed by the current experiment due to variable atmospheric conditions. Additionally, it indicates that there is a large degree of variability between different sites due to different atmospheric constituent abundances, and thermal and pressure gradients. NISM values are 1-5 times larger than such balloon measurements; however, the NISM filter has twice the bandwidth.

Rocket and satellite observations for similar wavelength and bandwidth filters give a good indication of the solar system zodiacal emission. The zodiacal emission results from sunlight scattered off interplanetary dust, and represents the limiting background radiation for any infrared astrophysical observations made from Earth or from a near-Earth orbit. Values obtained range from 3-8 $\mu \mathrm{Jy} \cdot \operatorname{arcsec}^{-2}$, but again are for bandwidths approximately half that of the NISM filter. The South Pole infrared sky background for the darkest sky conditions is only an order of magnitude above this limit, and represents the lowest value anywhere on Earth for near infrared observations. 


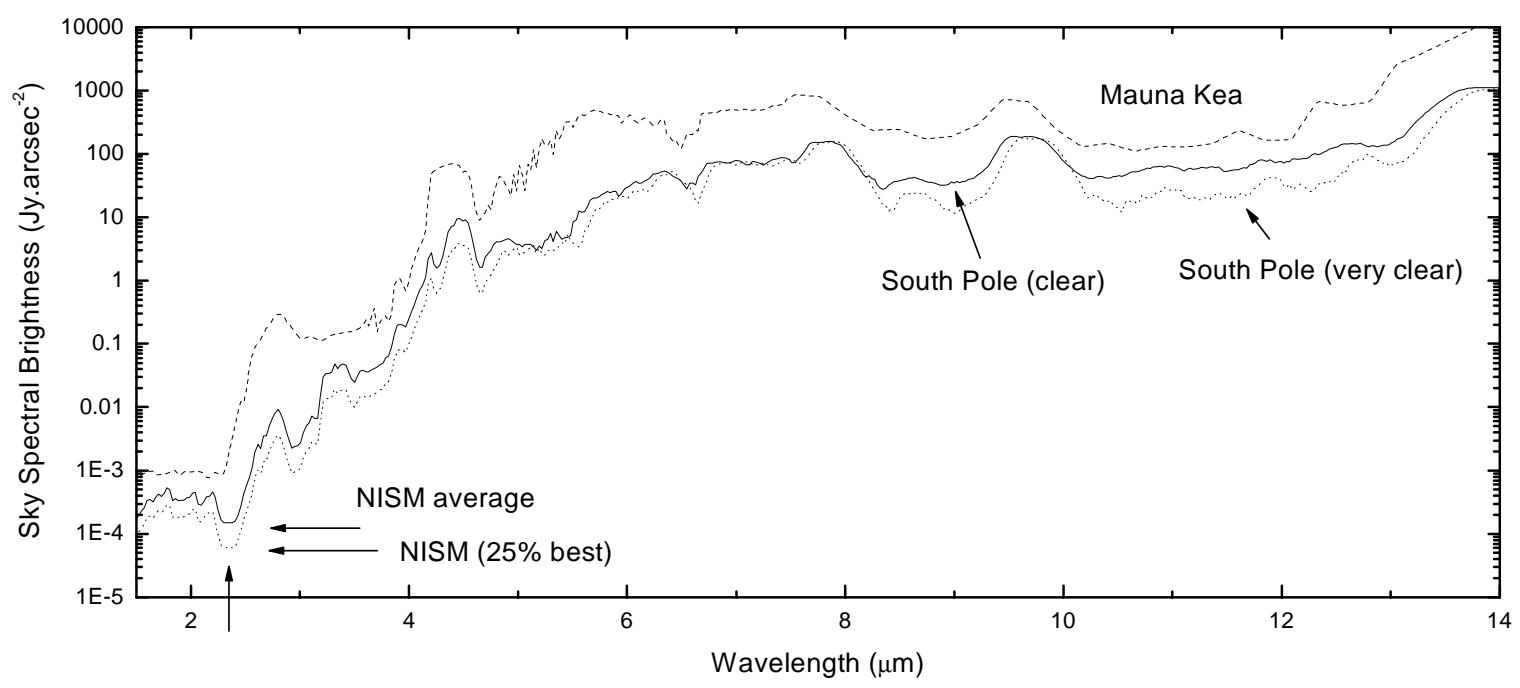

Figure 5. Sky spectral brightness in the near and mid infrared for the South Pole (in clear and very clear conditions) compared to Mauna Kea. South Pole data is from a combination of modelling and experimental results (see refs $(1,2)$ ). Mauna Kea data is from ref (5). The average and $25 \%$ best sky brightness values determined from the NISM are indicated in the figure.

\section{CONCLUSION}

The sky spectral brightness in the near infrared $\mathrm{K}_{\text {dark }}$ window at the South Pole has been determined from analysis of data collected by the Near Infrared Sky Monitor over the 2001 winter. It is found that the average winter-time sky flux is $\sim 220 \mu \mathrm{Jy} \cdot \operatorname{arcsec}^{-2}$. This falls to $80 \mu \mathrm{Jy} \cdot \operatorname{arcsec}^{-2}$ for the best $25 \%$ of conditions. These values compare well with those obtained from similar instruments operated previously at the South Pole. The data further support the proposition that the Antarctic plateau is the best site on Earth to perform near-infrared astronomy. The planned move of the NISM to Dome $\mathrm{C}$ on the Antarctic plateau in 2003 will further quantify the advantages obtainable to the astrophysical community by further developing the Antarctic plateau as an observatory site.

\section{ACKNOWLEDGEMENTS}

The authors would like to thank the US Center for Astrophysical Research in Antarctica (CARA) for support and collaboration throughout our site testing campaign. Logistic support by the US National Science Foundation (NSF), and funding from the Australian Research Council and the Australian Antarctic Division is gratefully acknowledged.

\section{REFERENCES}

1. A. Phillips, M. G. Burton, M. C. B. Ashley, J. W. V. Strorey, J. P. Lloyd, D. A. Harper, and J. Bally, "The near infrared sky emission at the South Pole in winter", Astrophys. J. 527, pp. 1009-1022, 1999.

2. M. A. Chamberlain, M. C. B. Ashley, M. G. Burton, A. Phillips, J. W. V. Storey, and D. A. Harper, "Mid-infrared observing conditions at the South Pole", Astrophys. J. 535, pp. 501-511, 2000.

3. J. W. V. Storey, M. C. B. Ashley, M. Boccas, M. A. Phillips, and A. E. T. Schinckel, "Infrared sky brightness monitors for Antarctica", Publ. Astron. Soc. Pac. 765, pp. 765-771, 1999.

4. J.S. Lawrence, M.C.B. Ashley, M.G. Burton, P.G. Calisse, J.R. Everett, R.J. Pernic, A. Phillips, and J.W.V. Storey, "Operation of the Near Infrared Sky Monitor at the South Pole", submitted to Publ. Astron. Soc. Aust., 2002.

5. P. Puxley, T. V. Hippel, and T. Geballe, "Observing constraints - sky background", Gemini Home Page, http://www.gemini.edu/sciops/ObsProcess/ObsProcIndex.html, 2001. 